\title{
GROWTH AND STRUCTURE OF BUFFER LAYERS FOR HIGH TEMPERATURE SUPERCONDUCTING FILMS
}

\author{
K. Fröhlich, A. Rosová, D. Machajdí, J. Šouc \\ Institute of Electrical Engineering, SAS, 84239 Bratislava, Slovakia \\ A. Figueras \\ Institut de Ciencia de Materials, CSIC, 08193-Bellaterra, Barcelona, Spain \\ F. Weiss, B. Chenevier \\ LMGP, ENSPG, BP 46, 38402 Saint Martin d'Heres, France \\ AND J. SNAUWAERT \\ Katholieke Univ. Leuven, Celestijnenlaan 200 D, 3001 Leuven, Belgium \\ We have studied thin $\mathrm{CeO}_{2}$ buffer layers prepared by aerosol MOCVD \\ on (1̄02) $\mathrm{Al}_{2} \mathrm{O}_{3}$ substrate at high deposition temperature, $T_{\mathrm{d}}=900^{\circ} \mathrm{C}$. A \\ texture analysis by $\mathrm{X}$-ray diffraction showed a high degree of epitaxial char- \\ acter of $\mathrm{CeO}_{2}$ films. A study of the microstructure by transmission electron \\ microscopy revealed that the $\mathrm{CeO}_{2}$ films are in a relaxed state being com- \\ posed of slightly misoriented blocks surrounded by dislocations. The films are \\ smooth, giving mean square root values of the surface roughness measured \\ by atomic force microscopy up to $1 \mathrm{~nm}$.
}

PACS numbers: $68.55 . \mathrm{Jk}$

\section{Introduction}

An intermediate buffer layer is necessary for the growth of high temperature superconducting (HTSC) thin films on technical substrates. R-plane sapphire ((1102) oriented) with $\mathrm{CeO}_{2}$ buffer layer seems to be an appropriate substrate for the growth of a whole family of ITSC thin films. An epitaxial character and smooth surface of the buffer layer are essential for the subsequent growth of HTSC films with a high critical current density. As an important lattice mismatch between the $\mathrm{CeO}_{2}$ film and the sapphire substrate $(5.7 \%$ and $13.7 \%$ along the [1101] and [1리이 directions, respectively) can give rise to structural imperfections, a careful control of the buffer layer microstructure is.necessary. In this paper we present microstructure and surface characterization of $(001)$ oriented $\mathrm{CeO}_{2}$ films grown on the (1102) $\mathrm{Al}_{2} \mathrm{O}_{3}$ substrate by the aerosol metal organic chemical vapor deposition (MOCVD) technique. 


\section{Sample preparation and characterization}

$\mathrm{CeO}_{2}$ films were prepared by the low pressure aerosol MOCVD process, described in details elsewhere [1]. As a precursor we have used heptafluorodimethyl octanodionate of $\mathrm{Ce}, \mathrm{Ce}(\mathrm{fod})_{3}$. Films with a thickness of $20 \mathrm{~nm}$ were deposited at the deposition temperature $T_{\mathrm{d}}=900^{\circ} \mathrm{C}$. The films were then characterized by a standard $\theta-2 \theta$ X-ray diffraction scans. The character of epitaxy of the films was studied by X-ray diffraction using the four circle Philips texture goniometer. A microstructure of the films was investigated by a transmission electron microscopy (TEM) using the JEOL JEM $1200 \mathrm{EX}$ electron microscope and a plane view technique. The atomic force microscopy (AFM) of the films surface was performed using the nanoscope III atomic force microscope in a tapping mode.

\section{Results and discussion}

The standard $\theta-2 \theta$ X-ray diffraction reveals that $\mathrm{CeO}_{2}$ films grow on $\mathrm{R}$-plane cut $\mathrm{Al}_{2} \mathrm{O}_{3}$ substrate with the preferred orientation $\mathrm{CeO}_{2}(001) \| \mathrm{Al}_{2} \mathrm{O}_{3}(1 \overline{1} 02)$. Some films contained a small amount of (111) oriented $\mathrm{CeO}_{2}$ grains in the (001) oriented $\mathrm{CeO}_{2}$ matrix. The growth of the (111) oriented grains can be stimulated by the substrate surface imperfections [2] or by instabilities of the deposition process. As it was observed by TEM a mean diameter of $40 \mathrm{~nm}$ is typical of the (111) oriented grains. An X-ray diffraction texture analysis of the (001) orient matrix revealed a high degree of the preferred orientation with FWHM value of the 002 reflection $\omega$-scan about $0.3^{\circ}$. The $\mathrm{CeO}_{2}$ films are in-plane aligned, as can be deduced from the fourfold modulated $\varphi$-scan of the $111 \mathrm{CeO}_{2}$ reflection, Fig. 1 . The FWHM of the $\varphi$-scan peak was equal to $1.4^{\circ}$, showing an excellent in-plane alignment of the deposited $\mathrm{CeO}_{2}$ film.

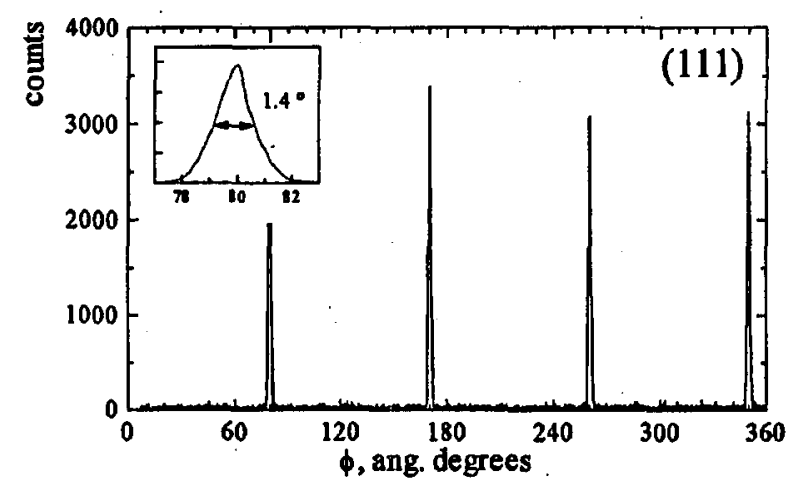

Fig. 1. $\varphi$-scan of the (111) $\mathrm{CeO}_{2} \mathrm{X}$-ray reflection.

Figure 2 shows a plane view TEM image of the $\mathrm{CeO}_{2}$ layer near the $\mathrm{CeO}_{2} /$ $\mathrm{Al}_{2} \mathrm{O}_{3}$ interface and its corresponding electron diffraction pattern. According to the diffraction pattern the layer is epitaxially grown with orientation $\mathrm{CeO}_{2}(001)$ [100] ॥ $\mathrm{Al}_{2} \mathrm{O}_{3}(01 \overline{1} 2)$ [2 $\left.\overline{11} 0\right]$. The electron diffraction exhibits a double diffraction effect and indicates that the $\mathrm{CeO}_{2}$ film is relaxed. 


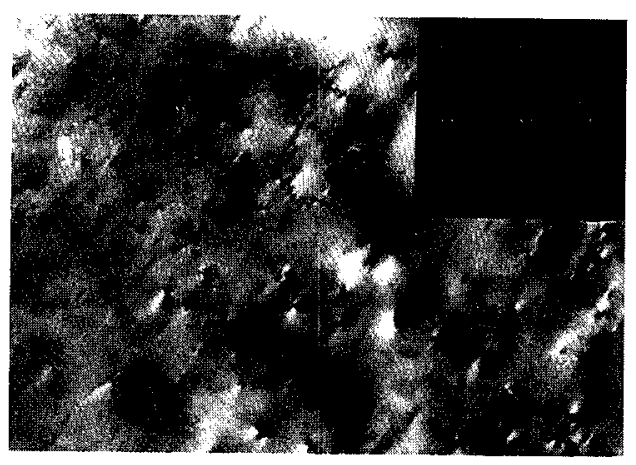

Fig. 2. TEM micrograph of the $\mathrm{CeO}_{2}$ film near the $\mathrm{CeO}_{2} / \mathrm{Al}_{2} \mathrm{O}_{3}$ interface. Selected area diffraction pattern exhibits a double diffraction effect.

a)
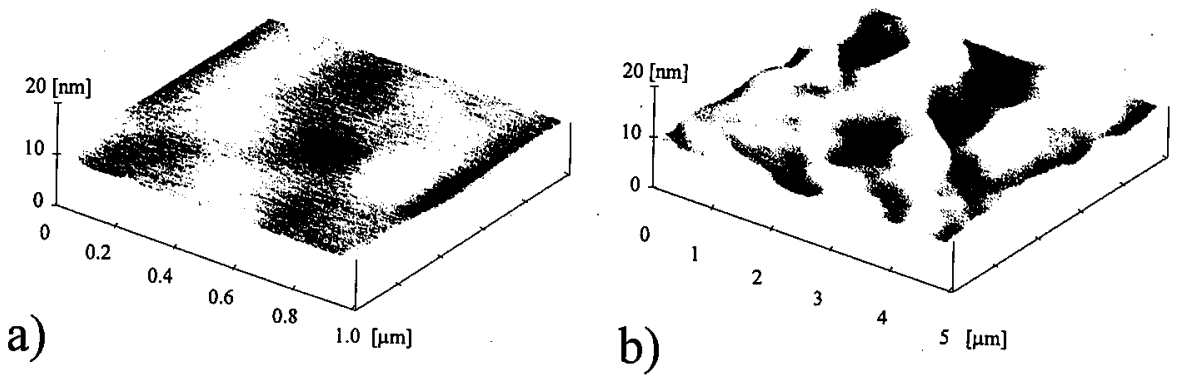

c)

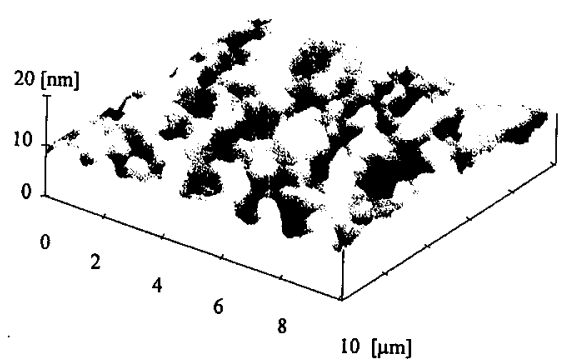

Fig. 3. AFM images of the $\mathrm{CeO}_{2}$ film with an area of (a) $1 \times 1 \mu \mathrm{m}^{2}$, (b) $5 \times 5 \mu \mathrm{m}^{2}$, (c) $10 \times 10 \mu \mathrm{m}^{2}$.

The epitaxial character of $\mathrm{CeO}_{2}$ layer is visualized also in TEM image by Moiré fringes which are an image interpretation of double diffraction in TEM (Fig. 2). The (001) oriented $\mathrm{CeO}_{2}$ layer consists of small blocks with the diameter of about $100 \mathrm{~nm}$, which are slightly rotated each to others around the film surface normal. The Moire fringes are parallel in the individual blocks and the mutual rotation of blocks is reflected by the magnified rotation of Moire fringes between the blocks. The mutual misorientation of the blocks deduced from the rotation of Moiré fringes gives values corresponding to the FWHM of $111 \varphi$-scan. The misorientation between blocks is a result of the substrate-film mismatch accommodation and is 
accompanied by a generation of dislocations parallel to the film surface normal. The (001) oriented matrix contains (111) oriented $\mathrm{CeO}_{2}$ grains with the mean diameter of $40 \mathrm{~nm}$.

The surface quality of the $\mathrm{CeO}_{2}$ films was examined by the atomic force microscopy. A set of AFM images with an area from 1 to $100 \mu \mathrm{m}^{2}$ is shown in Fig. 3. The surface of the film is smooth, giving mean square root values of the roughness $R_{q}=0.27,0.83$, and 0.95 for the $1,5 \times 5$, and $10 \times 10 \mu \mathrm{m}^{2}$ area, respectively. The value for the $1 \mu \mathrm{m}^{2}$ represents the mean value of 5 measurements performed at different places of the sample. The low value of $R_{q}$ for the $1 \mu \mathrm{m}^{2}$ area shows that at these regions the film is atomically smooth.

\section{Summary}

We have prepared epitaxial $\mathrm{CeO}_{2}$ films by aerosol MOCVD method on (1102) $\mathrm{Al}_{2} \mathrm{O}_{3}$ substrate. The films were grown at a high deposition temperature, $T_{\mathrm{d}}=900^{\circ} \mathrm{C}$. The films exhibit a high degree of epitaxy, as shown by the X-ray diffraction texture analysis. TEM observations reveal that the $\mathrm{CeO}_{2}$ films consist of small (about $100 \mathrm{~nm}$ diameter) slightly misoriented blocks. We sugrest that such a microstructure is generated due to the lattice mismatch strain between the film and the substrate. Double electron diffraction indicates relaxation of the $\mathrm{CeO}_{2}$ layer. The surface of the $\mathrm{CeO}_{2}$ films is smooth with $R_{q}$ up to $1 \mathrm{~nm}$. The $\mathrm{CeO}_{2}$ films prepared by aerosol MOCVD at a high deposition temperature are suitable as an intermediate buffer layer for the growth of epitaxial high- $T_{c}$ superconducting films.

This work was supported by the Slovak Scientific Grant Agency (grants VEGA 2/1087/95, 95/5305/107).

\section{References}

[1] K. Fröhlich, I. Souc, D. Machajdík, A.P. Kobzev, F. Weiss, J.P. Senateur, K.H. Dahmen, J. Phys. IV (France) C5 5, 533 (1995).

[2] Z. Lu, R. Iliskes, S.A. DiCarolis, A. Nel, R.K. Route, R.S. Feigelson, J. Cryst. Growth 156, 227 (1995). 